\title{
NOTE and activity in the skeletal muscle, liver, and brain of dogs
}

\author{
Shuichiro KANAI ${ }^{1)}$, Takuro SHIMADA ${ }^{1}$, Takanori NARITA $^{1 \text { ) }}$ and Ken OKABAYASHI ${ }^{1 \text { ** }}$ \\ ${ }^{1)}$ Department of Veterinary Medicine, College of Bioresource Sciences, Nihon University, 1866 Kameino, \\ Fujisawa, Kanagawa 252-0880, Japan
}

J. Vet. Med. Sci.

81(5): 712-716, 2019

doi: 10.1292/jvms.19-0049

Received: 23 January 2019

Accepted: 16 March 2019

Published online in J-STAGE: 26 March 2019
ABSTRACT. Phosphofructokinase-1 (EC:2.7.1.11, PFK-1) catalyzes the phosphorylation of fructose 6-phosphate to fructose 1,6-bisphosphate using adenosine triphosphate and is a key regulatory enzyme of glycolysis. Mammalian PFK-1 isozymes are composed of three kinds of subunits (PFK-M, $-L$, and -P), with different properties. It has been suggested that the proportion of PFK-1 subunits in different organs is based on the organ energy metabolism. In this study, we analyzed the activity and subunit composition of canine PFK-1. We found that, in dogs, the skeletal muscle only has PFK-M, the liver mainly has PFK-L, and the brain expresses all of them. The knowledge of the composition of PFK-1 could provide useful information for determination of the differences in glycolysis in various organs of dogs.

KEY WORDS: glycolysis, phosphofructokinase-1, subunit proportion

The efficiency of glucose catabolism varies because energetic substrates and the levels of energy required are diverse in different organs, tissues, or cells. The skeletal muscle at rest uses fatty acids as an energy source, but, at the time of exercise, activates glycolysis and consumes glucose, thereby using both aerobic and anaerobic respiration. Both glycolysis and glycogenesis occur in the liver to catabolize glucose for ATP production and release glucose into the blood at the time of hypoglycemia. The brain consumes a considerable amount of glucose and produces adenosine triphosphate (ATP) to maintain the membrane potential of neurons. Glycolysis is the basis of both anaerobic and aerobic respiration, and it occurs in nearly all organisms. Phosphofructokinase-1 (EC:2.7.1.11, PFK-1) catalyzes the phosphorylation of fructose 6-phosphate (F-6-P) to fructose 1,6-bisphosphate $\left(\mathrm{F}-1,6-\mathrm{P}_{2}\right)$ using ATP, and plays an important role as a key regulatory enzyme of glycolysis. In general, PFK-1 is sensitive to the energy level of the cell, as indicated by the abundance of ATP, and is regulated by a number of effectors including ATP, adenosine monophosphate (AMP), F-6-P, F-1,6- $\mathrm{P}_{2}$, and citrate. It has been suggested that the control of PFK-1 activity differs in each organ, tissue, or cell based on the performance of glucose catabolism. In mammals, tetrameric PFK-1 is composed of three subunits: muscle type (PFK-M), liver type (PFK-L), and platelet type (PFK-P, previously called C-type). The genes coding for these subunits are assigned to different chromosomes showing tissue-specific expression in humans [5]. In the skeletal muscle, the PFK-1 isozyme is composed only of PFK-M, but in the other tissues, all three subunits are expressed in varying proportions [5]. Several studies have shown that the various mammalian PFK-1 isozymes differ in their kinetic properties [2, 5, 8, 10, 11], together with the energy metabolism, which varies in each species depending on feeding behaviors and lifestyle. The PFK-1 composition in rats [3], rabbits $[6,8,11]$, cattle [7], and humans [5, 12] has been investigated by sodium dodecyl sulfate polyacrylamide gel electrophoresis (SDS-PAGE) using purified PFK-1 proteins. However, little is known about canine PFK-1. Therefore, in this study, we investigated the different canine PFK-1 in the skeletal muscle, liver, and brain, which organs had unique energy metabolism.

This study was approved by the Nihon University Animal Care and Use Committee (Permission number: AP13B074-1). Fresh organs were resected from four sacrificed healthy beagles ( 2 males and 2 females, 1 or 2 years old), and stored at $-80^{\circ} \mathrm{C}$ until the extraction. All the purification steps were carried out at $4^{\circ} \mathrm{C}$. Skeletal muscle, liver, and brain (300 $\mathrm{mg}$ each) resected using scissors were dissolved into $50 \mathrm{mM}$ Tris-phosphate buffer $(\mathrm{pH} 8)$ including $10 \mathrm{mM}$ dithiothreitol (DTT) and homogenized with a pellet mixer. The obtained homogenate was ultra-centrifuged at 100,000 $\times g$ for $30 \mathrm{~min}$. The supernatant was loaded onto PD-10 columns (GE Healthcare; Chicago, IL, U.S.A.) and gel filtration chromatography was performed to eliminate allosteric effectors of PFK-1. The solution obtained after gel filtration chromatography was used as the cytosolic protein solution, and the concentration of proteins in the solution was determined using the Bradford method [1], with bovine serum albumin as a standard.

The PFK-1 activity assay was conducted under optimal conditions unaffected by regulatory factors, as previously reported [7].

*Correspondence to: Okabayashi, K.: okabayashi.ken@nihon-u.ac.jp

O2019 The Japanese Society of Veterinary Science

This is an open-access article distributed under the terms of the Creative Commons Attribution Non-Commercial No Derivatives (by-nc-nd) License. (CC-BY-NC-ND 4.0: https://creativecommons.org/licenses/by-nc-nd/4.0/) 
Table 1. Primer sequences used in the quantitative PCR assays

\begin{tabular}{lclc}
\hline Symbol & GenBank accession no. & \multicolumn{1}{c}{ Primer sequences } & Length (bp) \\
\hline RPS18 & NM_001048082.1 & F: ATAGCCTTTGCCATCACAGCAATTA & 86 \\
& & R: TTGGTGAGATCGATGTCTGCTTTC & \\
PFK-M & NM_001003199.1 & $\begin{array}{l}\text { F: GCTGACACAGCCCTCAACACTATC } \\
\text { R: CGCAGTAGCCACCCATAGTTTC }\end{array}$ & 109 \\
& & F: AGACCTGAAAGCCAACGTGGAG & 173 \\
PFK-L & XM_544922.6 & R: GGCCCAGAACGTTGGTCCTA & \multirow{2}{*}{ PFK-P } \\
& XM_005617183.3 & F: GTCCAACGTGGAGCACCTGA & 122 \\
& & R: CCTCGGAGTACAGCTGGTAGATGAA & \\
\hline
\end{tabular}

The enzyme reaction was initiated by adding the cytosolic protein solution containing the enzyme at $25^{\circ} \mathrm{C}$, and the rate of $\mathrm{NADH}$ oxidation was monitored at $340 \mathrm{~nm}$ using a U-2900 spectrophotometer (Hitachi High-Technologies Corp., Tokyo, Japan). PFK-1 activity was determined in a reaction mixture containing the following components in a final volume of $1 \mathrm{ml}: 50 \mathrm{mM}$ HEPES buffer (pH 8.2), $100 \mathrm{mM} \mathrm{KCl}, 6.5 \mathrm{mM} \mathrm{MgCl}_{2}, 1 \mathrm{mM} \mathrm{NH}_{4} \mathrm{Cl}, 5 \mathrm{mM} \mathrm{KH}_{2} \mathrm{PO}_{4}, 0.3 \mathrm{mM} \mathrm{NADH}$, aldolase (0.5 units [U]), GDH (0.5 U), TPI (5 U), $1 \mathrm{mM} \mathrm{F-6-P,} 5 \mathrm{mM}$ ATP, and $0.1 \mathrm{mM}$ AMP. One unit of PFK-1 activity was defined as the amount of enzyme that phosphorylated $1 \mu \mathrm{mol}$ of $\mathrm{F}-6-\mathrm{P}$ per min at $25^{\circ} \mathrm{C}$.

Western blotting was performed using cytosolic protein extracts. Samples were boiled at $95^{\circ} \mathrm{C}$ for 5 min in SDS sample buffer (New England Biolabs; Ipswich, MA, U.S.A.). To apply the equal units of PFK-1 activity using the unpurified cytosolic protein samples obtained from various organs, the samples containing $0.3 \mathrm{mU}$ of PFK-1 were loaded in each lane of $7.5 \%$ MiniPROTEAN TGX gels (Bio-Rad Laboratories, Hercules, CA, U.S.A.) and separated by electrophoresis [4]. The separated proteins were transferred to polyvinylidene difluoride membranes, and treated with Block Ace (DS Pharma Biomedical, Osaka, Japan) for $50 \mathrm{~min}$. Thereafter, the membranes were incubated for $120 \mathrm{~min}$ with primary rabbit monoclonal antibodies (Abcam, Cambridge, U.K.) against PFK-M and -P (ab204131, 1:1,000), and against PFK-L (ab181064, 1:500). After washing, the membranes were incubated with horseradish peroxidase-conjugated anti-rabbit IgG (GE Healthcare, 1:10,000) for 90 min. Immunoreactivity was detected with the ECL Western blotting Analysis System (GE healthcare). The chemiluminescent signals of the membranes were measured, and densitometric analyses were performed using an ImageQuant LAS 4000 mini (GE Healthcare). The antibodies were stripped from the membranes using the Western Blot Stripping Buffer (Thermo Scientific, Rockford, IL, U.S.A.).

Total RNA was extracted from the samples of skeletal muscle, liver, and brain using TRIzol (Life Technologies Co., Carlsbad, CA, U.S.A.) according to the manufacturer's instructions. First-strand cDNA synthesis was performed using $500 \mathrm{ng}$ of total RNA with a PrimeScript RT Master Mix (Takara Bio Inc., Kusatsu, Japan). Quantitative PCR (qPCR) assays were performed using $2 \mu l$ of the first-strand cDNA in $25 \mu l$ of total reaction volume and SYBR Premix Ex Taq ${ }^{\mathrm{TM}}$ II (Takara Bio Inc.). The primers used in this study (Table 1) were purchased from Takara Bio Inc. PCRs were conducted using the Thermal Cycler Dice ${ }^{\circledR}$ Real Time System II TP900 (Takara Bio Inc.), and involved denaturation at $95^{\circ} \mathrm{C}$ for $5 \mathrm{sec}$ and annealing and extension at $60^{\circ} \mathrm{C}$ for 30 sec. The housekeeping gene was evaluated based on the standard deviation of the cycle threshold $(\mathrm{Ct})$ among $G A P D H, A C T B$, RPS18, and TBP using the BestKeeper software [9], and the results of GAPDH, ACTB, RPS18, and TBP were 1.15, 1.43, 0.69, and 1.34, respectively. Thus, the RPS18 was used for a standard to compare mRNA expression in skeletal muscle, liver, and brain in this study. Adequacy of primer sets used in this study was confirmed by the dissociation curve using the Thermal Cycler Dice ${ }^{\circledR}$ Real Time System Software Ver.5.11B (Takara Bio Inc.) and by agarose gel electrophoresis of the amplicons. PCR amplification efficiency was calculated from the slope of standard curve with serially diluted cDNA. qPCR results were calculated with Ct values derived by second derivative method using the Thermal Cycler Dice ${ }^{\circledR}$ Real Time System Software Ver.5.11B.

PFK-1 activity of the cytosolic solution deprived of its regulators was normalized to the protein concentration (Fig. 1). The PFK1 activity in the skeletal muscle, liver, and brain was $0.309 \pm 0.044$ (mean \pm standard error of the mean [SEM]), $0.011 \pm 0.001$ and $0.290 \pm 0.033 \mathrm{U} / \mathrm{mg}$ protein, respectively.

The results of western blotting assays are shown in Fig. 2. The detection sizes of the three subunits predicted were 86, 85, and $78 \mathrm{kDa}$ for PFK-P, -M, and -L respectively. In the same units of PFK-1, detection using the anti-PFK-M and -P antibody revealed a band as PFK-M in the skeletal muscle, a weak band as PFK-P in the liver, and two bands as PFK-P and -M in the brain, and detection using the anti-PFK-L antibody revealed a band in the liver and brain, but none in the skeletal muscle.

Upon analysis of the amplicons, we confirmed that they showed a single band in the agarose gel electrophoresis results and a single peak in the dissociation curve. The analysis of PCR amplification efficiency indicated that primers of RPS18 were amplified almost same in the cDNAs of skeletal muscle, liver, and brain (Fig. 3), and primers of each PFK subunit showed the comparable amplification efficiency (Fig. 4). Initial amounts of cDNA for RPS18 and PFK-1 subunits based from the qPCR results were shown in Fig. 5. Though the mRNA expression of RPS18 had little difference in skeletal muscle, liver, and brain, the total mRNA expression of the three subunits was high in the skeletal muscle and brain, and low in the liver. Skeletal muscle expressed $P F K-M$ strongly; the liver expressed $P F K-M$ and $-L$ at very low levels; and the brain expressed $P F K-M,-P$, and $-L$ in the descending order.

In our PFK-1 activity assay system under optimum conditions, the PFK-1 activity of the skeletal muscle and brain showed the highest activity, while the liver showed low activity. The PFK-1 activity under optimum conditions demonstrated maximum 


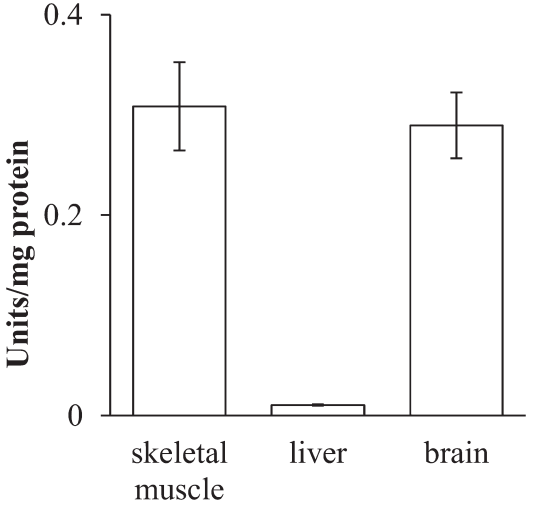

Fig. 1. PFK-1 activity in the cytosolic protein solution from the skeletal muscle, liver, and brain of dogs. Values were normalized to the protein concentration, and shown as the mean \pm SEM of 4 independent experiments.

skeletal muscle

$R^{2}=0.999$, efficiency $108.6 \%$

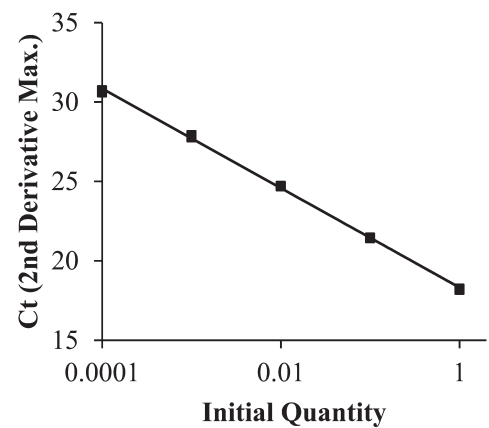

brain

$R^{2}=0.999$, efficiency $110.5 \%$

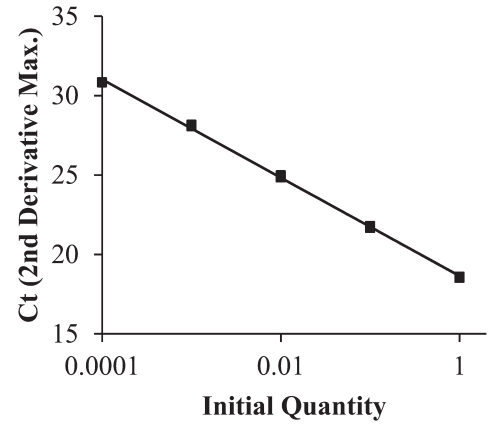

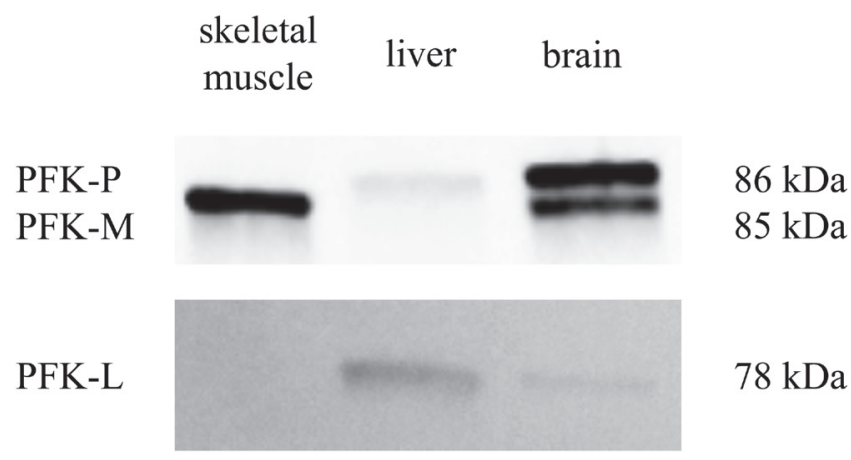

Fig. 2. PFK-1 subunit composition in canine skeletal muscle, liver, and brain. The respective PFK-1 subunits were detected by western blotting using primary antibodies against PFK-P, -M, and -L. The representative result in 4 independent experiments was shown.

liver

$R^{2}=0.999$, efficiency $109.7 \%$

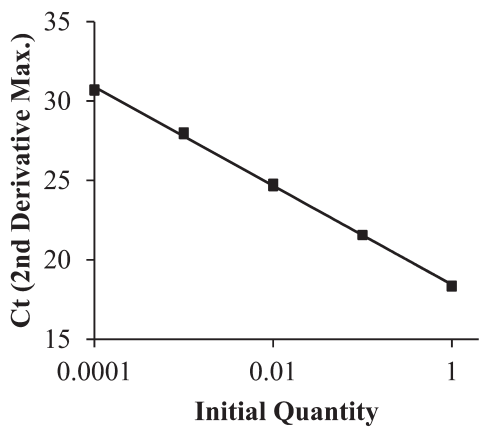

skeletal muscle, liver, and brain

$R^{2}=0.998$, efficiency $109.6 \%$

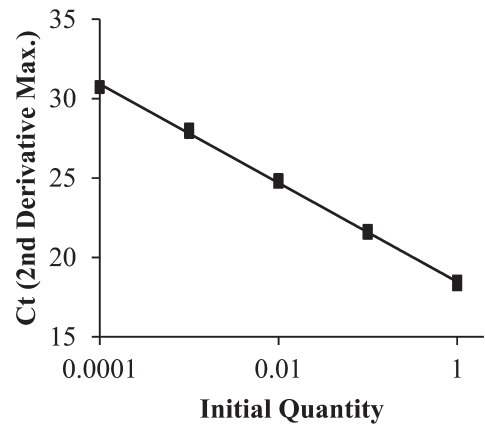

Fig. 3. PCR standard curve of internal standards. For internal standard RPS18, X-axes means serially diluted cDNA, and Y-axes means the cycle threshold values (2nd Derivative Maximum). PCR efficiencies calculated from slope of standard curves and the linear correlation coefiicient $\left(R^{2}\right)$ of standard curves.

velocity because of the insensitive to regulatory factors, and correlated with the amount of PFK-1. The PFK-1 activity in each organ would not demonstrate the maximal function under physiological conditions, due to the effect on regulatory factors.

Specifically, the PFK-1 activity in the skeletal muscle during exercise might demonstrate as high as that in the brain, while lower at rest. Interestingly, it was found that the total mRNA expression of the three subunits of PFK-1 in the skeletal muscle, liver, and brain correlated with the ratio of PFK-1 activity under optimal conditions. 
$P F K-M$

$R^{2}=0.994$, efficiency $111.1 \%$

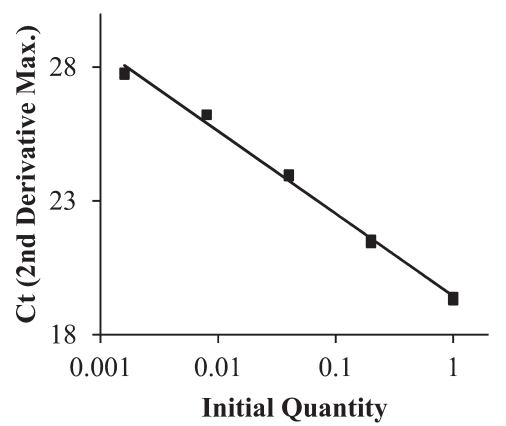

$P F K-P$

$R^{2}=0.997$, efficiency $105.3 \%$

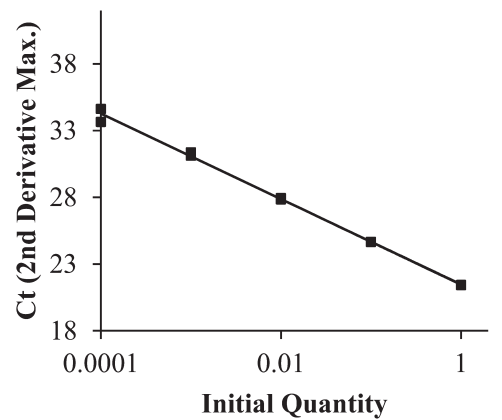

PFK-L

$R^{2}=0.991$, efficiency $120.1 \%$

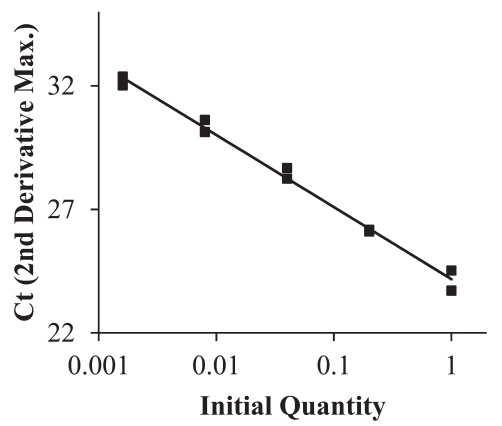

Fig. 4. PCR standard curve of target genes. For target genes PFK-M, -P, and -L, X-axes means serially diluted cDNA, and Y-axes means the cycle threshold values (2nd Derivative Maximum). PCR efficiencies calculated from slope of standard curves and the linear correlation coefiicient $\left(R^{2}\right)$ of standard curves.
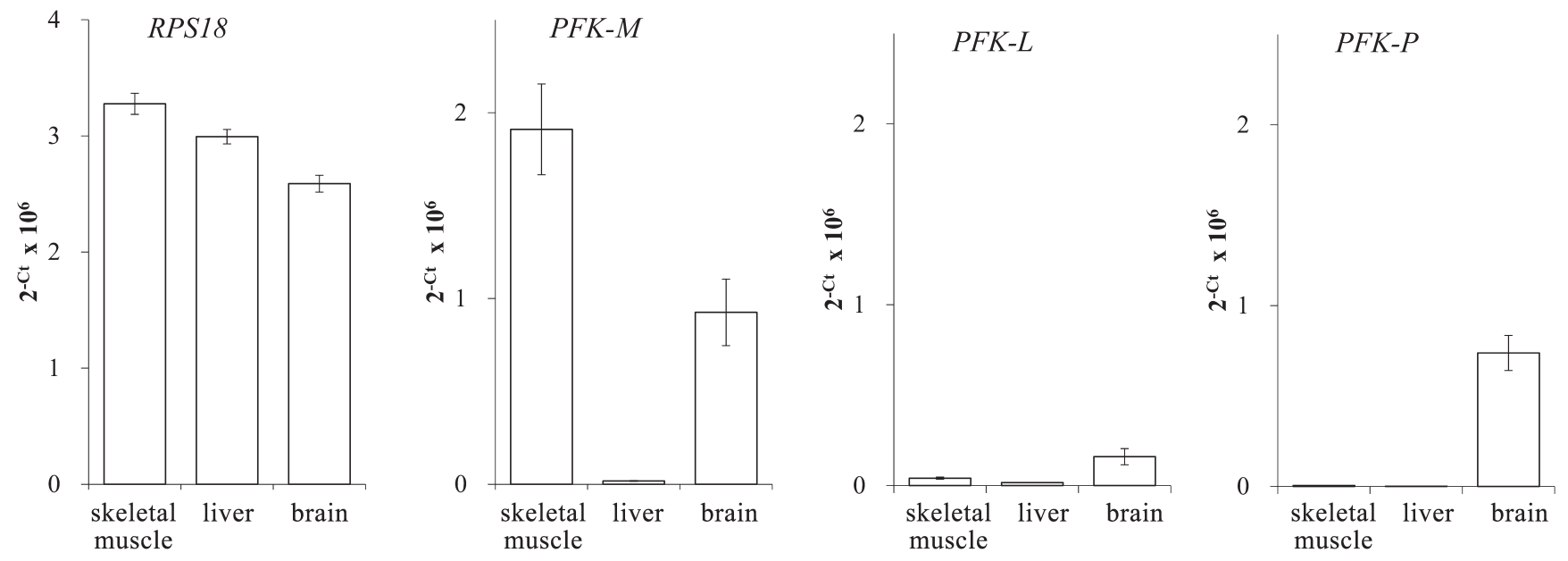

Fig. 5. Initial amounts of cDNA for RPS18 and PFK-1 subunits. The predicted mRNA expression of RPS18 and each PFK-1 subunit (PFK$\mathrm{M}$, -L, and -P) in the skeletal muscle, liver, and brain of dogs was calculated from Ct values. Results are shown as the mean \pm SEM of 4 independent experiments.

The skeletal muscle would express almost only the PFK-M subunit like other mammals, which is the one with the highest flexibility in terms of regulation; this allows adaptation of glycolysis in the skeletal muscle to different circumstances (rest or exercise).

The liver has low amounts of PFK-1 for glycolysis, as it also has the function of gluconeogenesis. Previous reports showed that the PFK-L, -M, and -P subunits of PFK-1 in human and rat livers are expressed in this descending order [4, 5], and that the rabbit and bovine livers mainly express the PFK-L subunit [7, 8]. Western blotting results for canine liver showed a dense band as PFK-L and a weak band as PFK-P. However, as per the qPCR results, the liver expressed very low levels of both PFK-M and -L. Thus, based on these results, it was suggested that the PFK-1 of canine liver mainly has the PFK-L subunit and possible existence of PFK-M and -P slightly. It is possible that liver PFK-1 composed by three kinds of subunits is the common features for omnivore and carnivore, but not for herbivore.

The PFK-1 of human and rat brains was composed of PFK-M (human: 55\%, rat: 49\%), PFK-P (human: 30\%, rat: 38\%), and PFK-L (human: $15 \%$, rat: 13\%) subunits [4, 5], and the PFK-1 of cattle contained the three subunits of PFK-1 [7]. The canine brain predominantly contained the PFK-M and -P subunits, but also contained PFK-L, based on the results of western blotting and qPCR assays. The composition of the three subunits of PFK-1 in the canine brain would be consistent with that in humans, rats, and cattle. One possibility is that the mammalian brain possesses all three subunits in order to maintain glycolysis under any conditions.

In summary, this study suggested that there would be the difference in liver PFK-1 subunit composition due to feeding behavior. It would provide useful information to elucidate the relation between the efficiency of canine glycolysis in each organ and PKF-1 composition. However, additional studies are necessary to investigate this phenomenon. 


\section{REFERENCES}

1. Bradford, M. M. 1976. A rapid and sensitive method for the quantitation of microgram quantities of protein utilizing the principle of protein-dye binding. Anal. Biochem. 72: 248-254. [Medline] [CrossRef]

2. Dunaway, G. A. 1983. A review of animal phosphofructokinase isozymes with an emphasis on their physiological role. Mol. Cell. Biochem. 52: 75-91. [Medline] [CrossRef]

3. Dunaway, G. A. and Kasten, T. 1986. Are the rat tissue/organ proportions of 6-phosphofructo-1-kinase subunits strain-specific? Biochem. J. 237: 157-161. [Medline] [CrossRef]

4. Dunaway, G. A. and Kasten, T. P. 1987. Nature of the subunits of the 6-phosphofructo-1-kinase isoenzymes from rat tissues. Biochem. J. 242: 667-671. [Medline] [CrossRef]

5. Dunaway, G. A., Kasten, T. P., Sebo, T. and Trapp, R. 1988. Analysis of the phosphofructokinase subunits and isoenzymes in human tissues. Biochem. J. 251: 677-683. [Medline] [CrossRef]

6. Foe, L. G. and Kemp, R. G. 1985. Isolation and characterization of phosphofructokinase C from rabbit brain. J. Biol. Chem. 260: 726-730. [Medline]

7. Fukushima, E. and Sugiya, H. 1992. Purification and characterization of phosphofructokinase in bovine parotid gland. Int. J. Biochem. 24: 1307-1314. [Medline] [CrossRef]

8. Kemp, R. G. 1971. Rabbit liver phosphofructokinase. Comparison of some properties with those of muscle phosphofructokinase. J. Biol. Chem. 246: 245-252. [Medline]

9. Okabayashi, K., Narita, T., Takashiro, S., Nadaoka, S., Kanai, S., Ito, D. and Kitagawa, M. 2019. mRNA expression of tumor-associated genes in canine grade I meningiomas. J. Vet. Med. Sci. 81: 369-372. [Medline] [CrossRef]

10. Sola-Penna, M., Da Silva, D., Coelho, W. S., Marinho-Carvalho, M. M. and Zancan, P. 2010. Regulation of mammalian muscle type 6-phosphofructo-1-kinase and its implication for the control of the metabolism. IUBMB Life 62: 791-796. [Medline] [CrossRef]

11. Tsai, M. Y. and Kemp, R. G. 1974. Rabbit brain phosphofructokinase. Comparison of regulatory properties with those of other phosphofructokinase isozymes. J. Biol. Chem. 249: 6590-6596. [Medline]

12. Vora, S., Wims, L. A., Durham, S. and Morrison, S. L. 1981. Production and characterization of monoclonal antibodies to the subunits of human phosphofructokinase: new tools for the immunochemical and genetic analyses of isozymes. Blood 58: 823-829. [Medline] 